\begin{tabular}{|c|c|c|}
\hline $\bar{E}$ & International Journal of Current Research in & \\
\hline P & Biosciences and Plant Biology & \\
\hline $\begin{array}{l}\text { EXCELLENT } \\
\text { PUBLISHERS }\end{array}$ & $\begin{array}{c}\text { ISSN: 2349-8080 (Online) • Volume } 3 \bullet \text { Number } 11 \text { (November-2016) } \\
\text { Journal homepage: } w \text { ww.ijcrbp.com }\end{array}$ & \\
\hline
\end{tabular}

\title{
Cloning and Expression Analysis of 3-Hydroxy-3-Methylglutaryl-CoA Reductase Gene from Matricaria chamomilla
}

\author{
Tingting Tao, Xiaomeng Liu, Jie Chang and Feng $\mathrm{Xu}^{*}$ \\ College of Horticulture and Gardening, Yangtze University, Jingzhou 434025, China \\ *Corresponding author.
}

\begin{abstract}
Chamomile (Matricaria chamomilla L.) is renowned for its production of essential oils, which major components are sesquiterpenoids. As the important enzyme in the sesquiterpenoid biosynthesis pathway, the enzyme 3-hydroxy-3-methylglutaryl-CoA reductase (HMGR) catelyzes the first committed step of isoprenoids biosynthesis in MVA pathway. In this study, a full-length cDNA encoding HMGR (designated as McHMGR, GenBank Accession No. KX925561) was successfully isolated from leaves of M. chamomilla by PCR. The full-length cDNA of McHMGR was 2276 bp long and contained a 1743 bp open reading frame (ORF) encoding a 580-amino-acid protein. The sequence of the McHMGR protein was highly homologous to those of HMGR proteins from other plant species. Phylogenetic tree analysis indicated that McHMGR shared the same ancestor in evolution with HMGRs and had a further relationship with other Dicotyledoneae species. qRT-PCR analysis revealed that the expression level of $M c H M G R$ is highest in the flowers and lowest in the stems. The isolation and characterization of McHMGR gene will be helpful to further study the role of McHMGR gene in the biosynthesis of sesquiterpenoids on M. chamomilla.
\end{abstract}

\section{Article Info}

Accepted: 12 October 2016

Available Online: 06 November 2016

\section{Ke y w o r d s}

Expression pattern

Matricaria chamomilla

McHMGR

Sesquiterpenoids

\section{Introduction}

Chamomile (Matricaria chamomilla L.) is one of the most predominant medicinal plants in Europe (Raal et al., 2012; Sayadi et al., 2014). The essential oil of chamomile flowers has significant antiphlogistic, spasmolytic and antimicrobial activity and is therefore used for several pharmaceutical, nutritional and cosmetic applications (Pirzad et al., 2006). M. chamomilla flowers contain 0.242.0 percent volatile oil that is blue in color. The two key constituents, a-bisabolol and chamazulene, account for 5065 percent of total volatile oil content. a-bisabolol, a sesquiterpene, has pharmacological actions and calming, carminative, and spasmolytic properties (Murti et al.,
2012; Son et al., 2014). In addition, it is a popular ingredient in topical health and beauty products for its soothing and anti-inflammatory effects on skin (Chadwick et al., 2013; Bonifacio et al., 2012; Su et al., 2015).

In nature, isoprenoids are synthesized from just two universal C5 precursors: isopentenyl diphosphate (IPP) and its isomer dimethylallyl diphosphate (DMAPP) (Attia et al., 2012; Pitera et al., 2007). Although IPP and DMAPP are the universal precursors for isoprenoid biosynthesis, there are two distinct pathways to biosynthesize isoprenoid in plant kingdom: the cytosolic mevalonate (MVA) pathway and the plastidic 2-Cmethyl-D-erythritol 4-phosphate (MEP) pathway 
(Vranová et al., 2013; Liu et al., 2006). The MVA pathway starting from 3 acetyl-CoA to finally yield IPP, is responsible for synthesizing sesquiterpenoids and sterols (Miziorko, 2011; Buhaescu and Izzedine, 2007). The MEP pathway producing IPP and DMAPP from pyruvate and D-glyceraldehyde 3-phosphate (GAP) is mainly responsible for forming monoterpenoids, diterpenoids constituents (Zhao et al., 2012).

The 3-hydroxy-3-methylglutaryl-Coenzyme A reductase (HMGR), which catalyzes the conversion of HMG-CoA to MVA, is a rate-limiting enzyme in isoprenoid biosynthesis via MVA pathway (Rui et al., 2012; Dai et al., 2011). In view of its significance in isoprenoid metabolism, genes encoding HMGRs have been isolated and extensively characterized from many plants including Withania somnifera (Akhtar et al., 2013), Coffea arabica (Pleschberger et al., 2011), Paris fargesii (Liang et al., 2014), Ginkgo biloba (Liao et al., 2015) and Camptotheca acuminata (Burnett et al., 1993). Here, we report for the first time the cloning and characterization of a new functional $H M G R$ gene from M. chamomilla. The expression profile of McHMGR in different organs is also described.

\section{Materials and methods}

\section{Plant materials}

Matricaria chamomilla was grown at $25 / 18^{\circ} \mathrm{C}$ in a controlled growth chamber (16 h light $/ 8 \mathrm{~h}$ dark). $M$. chamomilla leaves were collected from botanical garden at Yangtze University, and immediately placed in a $-80^{\circ} \mathrm{C}$ until use. Primer synthesis and DNA sequencing were performed by Shanghai Sangon Biotechnology Company, in China. Agarose Gel DNA extraction Kit Ver 4.0, pMD18-T vector kit, AMV Reverse Transcriptase, dNTPs, RNasin, and Taq DNA polymerase were purchased from Takara Company (Dalian, China).

\section{Cloning the full-length cDNA of $M c H M G R$}

Total RNA of $M$. chamomilla leaves was isolated using the TaKaRa MiniBEST Plant RNA Extraction kit (Dalian, China) according to the manufacturer's instructions. The extracted RNA was purified using oligo-dT-attached magnetic beads. The purified mRNA was cleaved into small pieces by adding fragmentation buffer. Cleaved mRNAs were used as templates to construct RNA-seq library according to the manufacturer's protocol. The transcriptome sequencing libraries were generated using a NEB Next ${ }^{\circledR}$ Ultra $^{\mathrm{TM}}$
RNA Library Prep Kit for Illumina ${ }^{\circledR}$ (NEB, USA). Sequencing run was performed at Biomark Biomarker Co., Beijing, China using Illumina Hiseq 2500 platform. The assembled unigenes were searched against the $\mathrm{Nr}$ and $\mathrm{Nt}$ databases, and the Swiss-Prot protein and COG/KOG databases using BLAST with an cutoff Evalue of $1 \mathrm{e}^{-5}$. To assign functional annotations, the unigenes were searched against Pfam using HMMER 3.0 (Finn et al., 2011) with E-value of $1 \mathrm{e}^{-2}$. Based on the annotation information of the unigenes of $M$. chamomilla, a pair of specific primers (McHMGRu: 5'ATGGATGTCCGACGAGGATC-3' and McHMGRd: 5'-TCCACCAGAGATCTTGCTTCC-3') were designed and synthesized according to the sequence of HMGR unigene. Single strand cDNA was synthesized using PrimeScript cDNA Synthesis Kit (TaKaRa, Japan). McHMGR cDNA was amplified with the one-step RTPCR kit (Dalian TaKaRa, Dalian, China) under the following conditions: $94^{\circ} \mathrm{C}$ for $3 \mathrm{~min}$, followed by 32 cycles of amplification at $94^{\circ} \mathrm{C}$ for $1 \mathrm{~min}, 54^{\circ} \mathrm{C}$ for $30 \mathrm{~s}$, and $72^{\circ} \mathrm{C}$ for $1 \mathrm{~min}$; and extension for $10 \mathrm{~min}$ at $72^{\circ} \mathrm{C}$. The PCR product was purified and cloned into the pMD18-T vector, and transformed into Escherichia coli strain DH5a followed by sequencing.

\section{Bioinformatics and molecular evolution analysis}

Sequence assembly was performed with programs of DNAstar (http://www.dnastar.com). The nucleotide sequence, deduced amino acid sequence and open reading frame (ORF) were analyzed, and the sequence comparison was conducted through database search (http://www.ncbi.nlm.nih.gov). The calculated isoelectric point (pI) and molecular weight of the McHMGR protein were computed with the software of Compute $\mathrm{pI} / \mathrm{Mw}$ Tool at http://web.expasy.org/compute_pi/. Multiple sequence alignment was performed with the software Vector NTI 11.5 program. Phylogenetic analysis of McHMGR and other HMGR from other plants were aligned with CLUSTALX 2, and subsequently, a phylogenetic tree was constructed by the neighbor-joining (NJ) method with MEGA 6 software (Kumar et al., 2001).

\section{Differential expression analysis of McHMGR by qRT- PCR}

The expression level of McHMGR was determined by realtime PCR (qRT-PCR). Aliquots of $1 \mu \mathrm{g}$ total RNA was used as the template for qRT-PCR. qRT-PCR was performed using a Bio-Rad Mini Opticon ${ }^{\mathrm{TM}}$ Real-time PCR Mini Cycler (BioRad, Hercules, CA, USA) with SYBR Premix Ex Taq ${ }^{\mathrm{TM}}$ II Kit (Dalian TaKaRa) according to the 
method of Xu et al. (2014). The primers for McHMGR (McHMGRu: 5'-GCTTCACAATCTGCTTGCCTAA-3', McHMGRd: 5'-TTGACGAGTTGTCCTGCTGAAA-3') and referential gene 18S rRNA gene (18SU: 5'ACCGAGCGTCGAGTGGATTAA-3' and 18SD: 5'CTAGTTCGTGCGTCCGTCAAA-3') were designed using the Sequence Detection System software. Raw data were analyzed with MiniOpticon ${ }^{\mathrm{TM}}$ Real-time PCR Detection system, and expression level was normalized into $18 \mathrm{~S}$ gene to minimize the variation in the cDNA template levels. qRT-PCR data were technically replicated with error bars, representing mean $\pm \mathrm{SD}(\mathrm{n}=3)$. The relative expression fold of each sample was calculated by its $\mathrm{Ct}$ value normalized to the $\mathrm{Ct}$-value of reference gene using the $2^{-\Delta \Delta \mathrm{Ct}}$ method described by Livak and Schmittgen (2001).

\section{Results}

\section{Cloning and characterization of the full-length cDNA of $M c H M G R$ gene}

A total of 82,946 unigenes have been identified in $M$. chamomilla RNA-Seq database through annotation against public protein databases (data not published). Among these unigenes, one HMGR member was identified based on the annotation information. Using a pair of specific primers based on the HMGR unigene (Gene ID: McBMK_36655) of transcriptome data, we performed PCR using cDNA as the template, and a 2276-bp fragment was amplified. Using an RT-PCR method, a cDNA fragment encoding $H M G R$, designated as McHMGR (GenBank Accession No. KX925561), was isolated and characterized. The nucleotide sequence of $M c H M G R$ had high similarity with $H M G R$ genes of other plants (Table 1). The results indicate that the gene we cloned is a member of the HMGR gene family. As shown in Fig. 1, McHMGR cDNA contained a $1743 \mathrm{bp}$ open reading frame (ORF) encoding a 580 amino acid protein.

Table 1. Nucleotide sequence of $M c H M G R$ similarity to the $H M G R$ genes of other plant species.

\begin{tabular}{lll}
\hline Species & Accession no. & $\begin{array}{l}\text { Homology } \\
(\%)\end{array}$ \\
\hline Taraxacum kok-saghyz & HQ857601.1 & 81 \\
Ligularia fischeri & DQ916106.1 & 84 \\
Camellia sinensis & KF649853.1 & 78 \\
Prunus mume & XM_008237609.2 & 77 \\
Gossypium hirsutum & XM_016860153.1 & 79 \\
Glycine max & XM_003534178.3 & 77 \\
\hline
\end{tabular}

\section{1}

61

121

181

61

241

301

301
101

361

121

421

141
481

161

541

181

601

201

221

721

241

781

261

841
281

901

301

961

321

102

341
1081

361

1141

381

1201

401

1261

421

1321

441

1381

461

144

481
1501

501

1561

521

1621

541

1681

561

1741

581

1801

601

1861

621

1921

641

1981

2041

2101

2161

2221
CTAACTGTATACAAGATTCAAATCAAGCGTGTAGTTATCCATATACCTATAAATAAAGCC ACCATATTTACAACCATTTTGCAAATAAGCTACTTTACAAATCCCATTATCTGTATTCGT TCATCCTCAACGAACTCGTCTTCGGCTCCATCCCATCTACCCAGTAGTACTACCGTAGCA AAAGGGATGGATGTCCGACGAGGATCTTCGTTGAAACCTTCTTTTAAGAATCATGTTTCA \begin{tabular}{lllllllllllllllllll}
\hline$M$ & $D$ & $V$ & $R$ & $R$ & $G$ & $S$ & $S$ & $L$ & $K$ & $P$ & $S$ & $F$ & $K$ & $N$ & $H$ & $V$ & $S$
\end{tabular} GATGATAATAAGATGTTTGAAGCCAAGGACAAAAAAGTGGTGGACGGTTCTGTCCCACTT

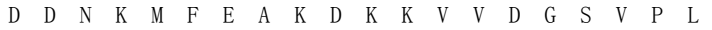
TCTTTAGGGATTTCAAATGGTGTTTTCTTTACTGTTTTCTTTTCGGTTACGTATTTTCTT $\begin{array}{llllllllllllllllllll}S & L & G & I & S & N & G & V & F & F & T & V & F & F & S & V & T & Y & F & L\end{array}$ CTTACTAGATGGCGTGAAAAGATCCGTAACTCGACTCCACTCCATGTTGTCACCATGTCG $\begin{array}{llllllllllllllllllll}\mathrm{L} & \mathrm{T} & \mathrm{R} & \text { W } & \mathrm{R} & \mathrm{E} & \mathrm{K} & \mathrm{I} & \mathrm{R} & \mathrm{N} & \mathrm{S} & \mathrm{T} & \mathrm{P} & \mathrm{L} & \mathrm{H} & \mathrm{V} & \mathrm{V} & \mathrm{T} & \mathrm{M} & \mathrm{S}\end{array}$ GAGATCGCTGCAATCTTCTTGTTTGTGGCGTCGTTTATCTACCTTATTGGCTTCTTTGGG

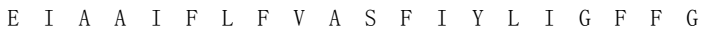
ATGAGTTTTGTTAACCCTACGCCTTATTCAGACGATGAAGAAGAAGAGGAGATAGAAGGT $\begin{array}{llllllllllllllllllll}M & S & F & V & N & P & T & P & Y & S & D & D & E & E & E & E & E & I & E & G\end{array}$ GACGTGAACGAAATTGTGCGTAAGGAAGACACTCGTGTGACCTCTTGTGGTGCAGCCCTA $\begin{array}{llllllllllllllllllll}D & V & N & E & I & V & R & K & E & D & T & R & V & T & S & C & G & A & A & L\end{array}$ GACTGTGAGTCAGATGTGGTCGTAAAACACGTTATAAAGAAAGACTTGGAGCATTTACCA

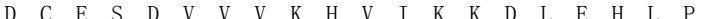
ACTGAAACAGTTCTTACGGAAGAGGATGAGGAGGTAATAAAAGCGGTTGTTTCGGGTAAA $\begin{array}{llllllllllllllllllll}\mathrm{T} & \mathrm{E} & \mathrm{T} & \mathrm{V} & \mathrm{L} & \mathrm{T} & \mathrm{E} & \mathrm{E} & \mathrm{D} & \mathrm{E} & \mathrm{E} & \mathrm{V} & \mathrm{I} & \mathrm{K} & \mathrm{A} & \mathrm{V} & \mathrm{V} & \mathrm{S} & \mathrm{G} & \mathrm{K}\end{array}$ ACACCATCTTACTCGTTGGAATCCAAGCTTGGGGATTGTAAACGTGCCGCTTTCATAAGG $\begin{array}{lllllllllllllllllllll}\mathrm{T} & \mathrm{P} & \mathrm{S} & \mathrm{Y} & \mathrm{S} & \mathrm{L} & \mathrm{E} & \mathrm{S} & \mathrm{K} & \mathrm{L} & \mathrm{G} & \mathrm{D} & \mathrm{C} & \mathrm{K} & \mathrm{R} & \mathrm{A} & \mathrm{A} & \mathrm{F} & \mathrm{I} & \mathrm{R}\end{array}$ CGAGTAGCCCTGGAGAGGATTACTGGGAAATCTCTTGATGGTTTGCCTCTAGAAGGGTTT $\begin{array}{llllllllllllllllllll}R & V & A & \text { L } & \text { E } & R & \text { I } & \text { T } & G & \text { K } & \text { S } & \text { L } & \text { D } & G & \text { L } & \text { P } & \text { L } & \text { E } & G & \text { F }\end{array}$ GATTACGAATCGATATTGGGACAGTGTTGTGAGATGCCAGTTGGTTATGTTCAAATACCC $\begin{array}{llllllllllllllllllll}D & \text { Y } & \text { E } & \text { S } & \text { I } & \text { L } & G & \text { Q } & \text { C } & \text { C } & \text { E } & \text { M } & \text { P } & \text { V } & \text { G } & \text { Y } & \text { V } & \text { Q } & \text { I } & \text { P }\end{array}$ GTTGGTATTGCTGGTCCGATGTTGTTGGATGGACAGGAGTTTACCGTGCCCATGGCGACT

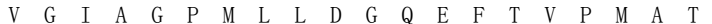
ACTGAAGGGTGTCTTGTGGCTAGTACCAATAGGGGTTGTAAGGCCATTTATGTATCTGGT $\begin{array}{llllllllllllllllllll}\mathrm{T} & \mathrm{E} & \mathrm{G} & \mathrm{C} & \mathrm{L} & \mathrm{V} & \mathrm{A} & \mathrm{S} & \mathrm{T} & \mathrm{N} & \mathrm{R} & \mathrm{G} & \mathrm{C} & \mathrm{K} & \mathrm{A} & \mathrm{I} & \mathrm{Y} & \mathrm{V} & \mathrm{S} & \mathrm{G}\end{array}$ GGTGCAACTAGTGTCCTACTTAAAGATGGCATGACTCGAGCTCCGGTTGTTAGGTTTGGA

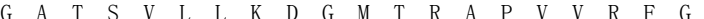
ACCGCGAAGAGGGCTGCTGATTTGAAGTTCTTCTTGGAGGAACCACTCAACTTTGATACA $\begin{array}{llllllllllllllllllll}\mathrm{T} & \mathrm{A} & \mathrm{K} & \mathrm{R} & \mathrm{A} & \mathrm{A} & \mathrm{D} & \mathrm{L} & \mathrm{K} & \mathrm{F} & \mathrm{F} & \mathrm{L} & \mathrm{E} & \mathrm{E} & \mathrm{P} & \mathrm{L} & \mathrm{N} & \mathrm{F} & \mathrm{D} & \mathrm{T}\end{array}$ CTTGCATCTGTTTTCAACAAATCAAGCCGATTTGGGAGGCTTCAGAGAATTCAATGTGCG $\begin{array}{llllllllllllllllllll}\mathrm{L} & \mathrm{A} & \mathrm{S} & \mathrm{V} & \mathrm{F} & \mathrm{N} & \mathrm{K} & \mathrm{S} & \mathrm{S} & \mathrm{R} & \mathrm{F} & \mathrm{G} & \mathrm{R} & \mathrm{L} & \mathrm{Q} & \mathrm{R} & \mathrm{I} & \mathrm{Q} & \mathrm{C} & \mathrm{A}\end{array}$ ATAGCCGGGAAGAATCTGTACGTAAGGTTTACATGCAGCACTGGTGATGCAATGGGGATG $\begin{array}{lllllllllllllllllllll}I & A & G & K & N & L & Y & V & R & F & T & C & S & T & G & D & A & M & G & M\end{array}$ AACATGGTTTCCAAAGGTGTTCAAAATGTTCTAGACTATCTCCAAGCTGATTTCCCCGAC $\begin{array}{llllllllllllllllllll}N & M & V & S & K & G & V & Q & N & V & \text { L } & \text { D } & \text { Y } & \text { L } & \text { Q } & \text { A } & \text { D } & \text { F } & \text { P } & \text { D }\end{array}$ ATGGACGTCATTGGCATATCTGGAAACTATTGTTCGGATAAGAAACCGGCAGCGGTGAAT $\begin{array}{llllllllllllllllllll}M & D & V & I & G & I & S & G & N & Y & C & S & D & K & K & P & A & A & V & N\end{array}$ TGGATAGAAGGGAGAGGTAAATCAGTGGTGTGTGAGGCAATCATAAAGGAAGAAATAGTG

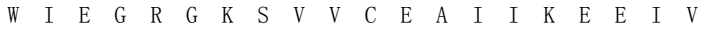
AAAAAGGTATTGAAAACAACTGTAGCTTCCTTGGTCGAACTGAACATGCTCAAGAACCTC

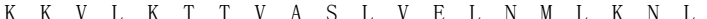
ACGGGATCCGCTATGGCTGGTGCTCTTGGTGGCTTCAACGCGCATGCAAGTAACATCGTG $\begin{array}{llllllllllllllllllll}T & G & S & A & M & A & G & A & \text { L } & G & G & F & N & A & H & A & S & N & I & V\end{array}$ TCAGCTGTGTTCCTTGCTACTGGACAAGATCCAGCTCAGAACGTCGAAAGCTCTCATTGT $\begin{array}{llllllllllllllllllll}S & A & V & F & \text { L } & \text { A } & \text { T } & G & \text { Q } & \text { D } & \text { P } & \text { A } & \text { Q } & \text { N } & \text { V } & \text { E } & \text { S } & \text { S } & \text { H } & \text { C }\end{array}$ ATCACCATGATGGAAGCTGTGAACGATGGCAAAGACCTTCACGTGTCTGTGACCATGCCA $\begin{array}{llllllllllllllllllll}\text { I } & \mathrm{T} & \mathrm{M} & \mathrm{M} & \mathrm{E} & \mathrm{A} & \mathrm{V} & \mathrm{N} & \mathrm{D} & \mathrm{G} & \mathrm{K} & \mathrm{D} & \mathrm{L} & \mathrm{H} & \mathrm{V} & \mathrm{S} & \mathrm{V} & \mathrm{T} & \mathrm{M} & \mathrm{P}\end{array}$ TCAATTGAGGTTGGGACTGTGGGTGGTGGAACTCAGTTGGCTTCACAATCTGCTTGCCTA $\begin{array}{llllllllllllllllllll}S & I & E & V & G & T & V & G & G & G & T & Q & \text { L } & \text { A } & \text { S } & \text { Q } & \text { S } & \text { A } & \text { C } & \text { L }\end{array}$ AACTTGTTGGGAGTGAAGGGTGCAAACAAAGAACTTGCTGGATCAAATGCCCGACAATTG $\begin{array}{llllllllllllllllllll}N & L & L & G & V & K & G & A & N & K & E & L & A & G & S & N & A & R & Q & L\end{array}$ GCTAAGGTTGTCGCAGCCGCAGTTCTAGCTGGAGAGTTATCTCTCATGTCGGCAATTTCA $\begin{array}{llllllllllllllllllll}\text { A } & \text { K } & \text { V } & \text { V } & \text { A } & \text { A } & \text { A } & \text { V } & \text { L } & \text { A } & \text { G } & \text { E } & \text { L } & \text { S } & \text { L } & \text { M } & \text { S } & \text { A } & \text { I } & \text { S }\end{array}$ GCAGGACAACTCGTCAAAAGTCACATGAAATACAACCGCTCCACCAGAGATCTTGCTTCC $\begin{array}{llllllllllllllllllll}A & G & Q & L & V & K & S & H & M & K & Y & N & R & S & T & R & D & L & A & S\end{array}$ AAGGCTTAAGCTTCTCTCGATTTGTTTGTTGTCATATTGGAGGGCAGCTATCAGTTATTC $\mathrm{K} \quad \mathrm{A} *$

TTGTTTCTACTGGTCTTTTTGAGCTCTTGTTCTTCCACTGATCTTCGTTCAGCTCTTGGT TGTGAGCAATTCGAGTTTAAGGTTCAAATATATACTATACCTTAAGTATTTGAAGTGCAT GTTAAGCATTTGGTATATGAATAATAAAGTGTTTAATAATTAGCAATACGTGTGATTGCG ATTGTAAATGACTATCAAAATCAAGTATTGTCGCACATTAAGCTCTAATTTGGTCCCATT TTTGTAGTCTAATCTACAAATAGGCGTAAAAGATATACAAAATTGATGGTTTCAAG

1: The nucleotide acid sequence and deduced amino acid sequence of McHMGR. The underline for specific primers McHMGRu and McHMGRd.

\section{Characterization of the deduced McHMGR protein}

By using the software of Compute $\mathrm{pl} / \mathrm{Mw}$ Tool, the calculated isoelectric point (pI) and molecular weight of the McHMGR were predicted to be 5.90 and $62.4 \mathrm{kDa}$, 
respectively. A database search with BlastP and multialignment by Vector NTI 11.5, results showed that McHMGR exhibited high similarity to HMGR proteins from other plants (Fig. 2). The deduced McHMGR protein sequence showed $83,86,75,75,75$ and $77 \%$ identities to the counterparts of Cynara cardunculus var. scolymus, Taraxacum kok-saqhyz, Sesamum indicum, Gossypium raimondii, Prunus meme, Ricinus communis, thereby indicating that McHMGR belongs to plant HMGR superfamily.
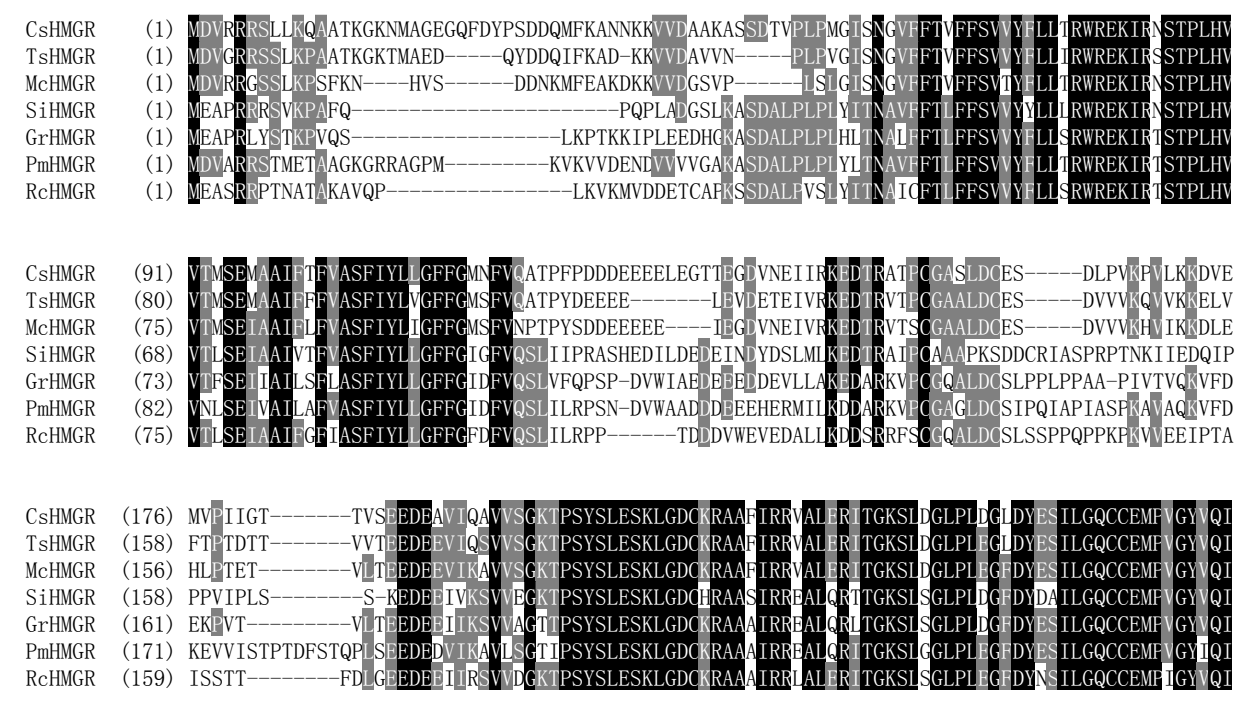

RcHMGR (159) ISSTT---_----FDLGEEDEEIIRSVVDGKTPSYSLESKLGDCKRAAAIRRLALERITGKSLSGLPLEG DYNSILGQCCEMF IGYVQ
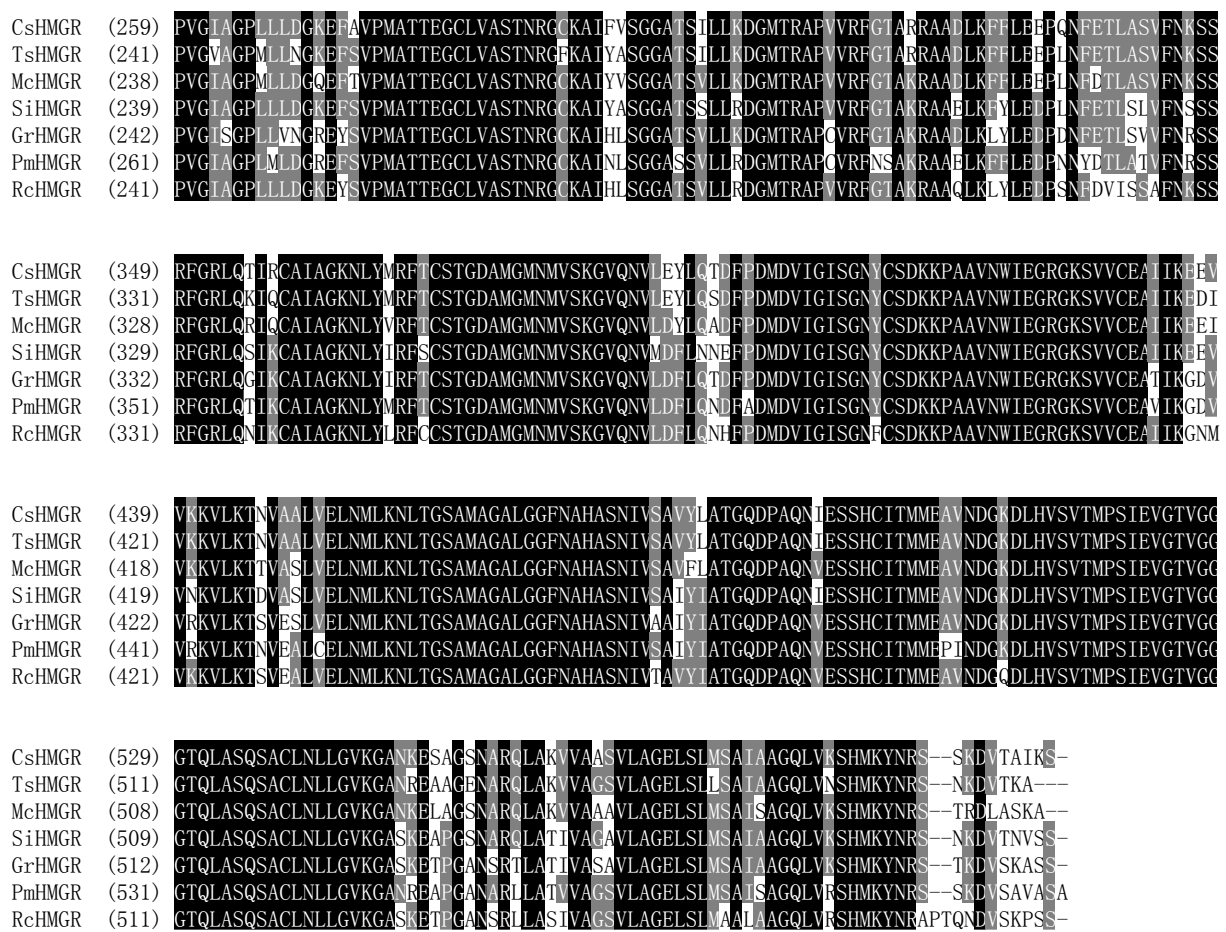

Fig. 2: Multiple alignment of amino acid sequences of McHMGR along with the selected HMG-CoA reductases. The completely identical amino acids are indicated with white foreground and black background. The conserved amino acids are indicated with black foreground and grey background. Non-similar amino acids are indicated with black foreground and white background. The species, protein names and GenBank accession numbers are following: Cynara cardunculus var. scolymus: CsHMGR (KVI04181.1); Taraxacum kok-saqhyz: TsHMGR (AEA92686.1); Sesamum indicum: SiHMGR (XP_011092919.1); Gossypium raimondii: GrHMGR (XP_012479905.1); Prunus meme: pmHMGR (XP_008235831.1); Ricinus communis: RcHMGR (XP_002514400.1).

T. Tao et al. (2016) / Cloning and Expression Analysis of 3-Hydroxy-3-Methylglutaryl-CoA Reductase Gene from 


\section{Molecular evolution analysis}

To investigate the evolutionary relationships among McHMGR and HMGR proteins from other plants, the phylogenetic tree was constructed using neighborjointing method. As shown in Fig. 3, McHMGR belonged to Dicotyledoneae in the branch of
Dicotyledoneae, Monocotyledoneae and Gymnospermae. The McHMGR protein has the closest relationship to LfHMGR and TsHMGR of Asteraceae. The results suggest that McHMGR shares a common evolutionary with other plant HMGR proteins based on conserved structure and sequence characteristics, such as amino acid homologies and conserved motifs.

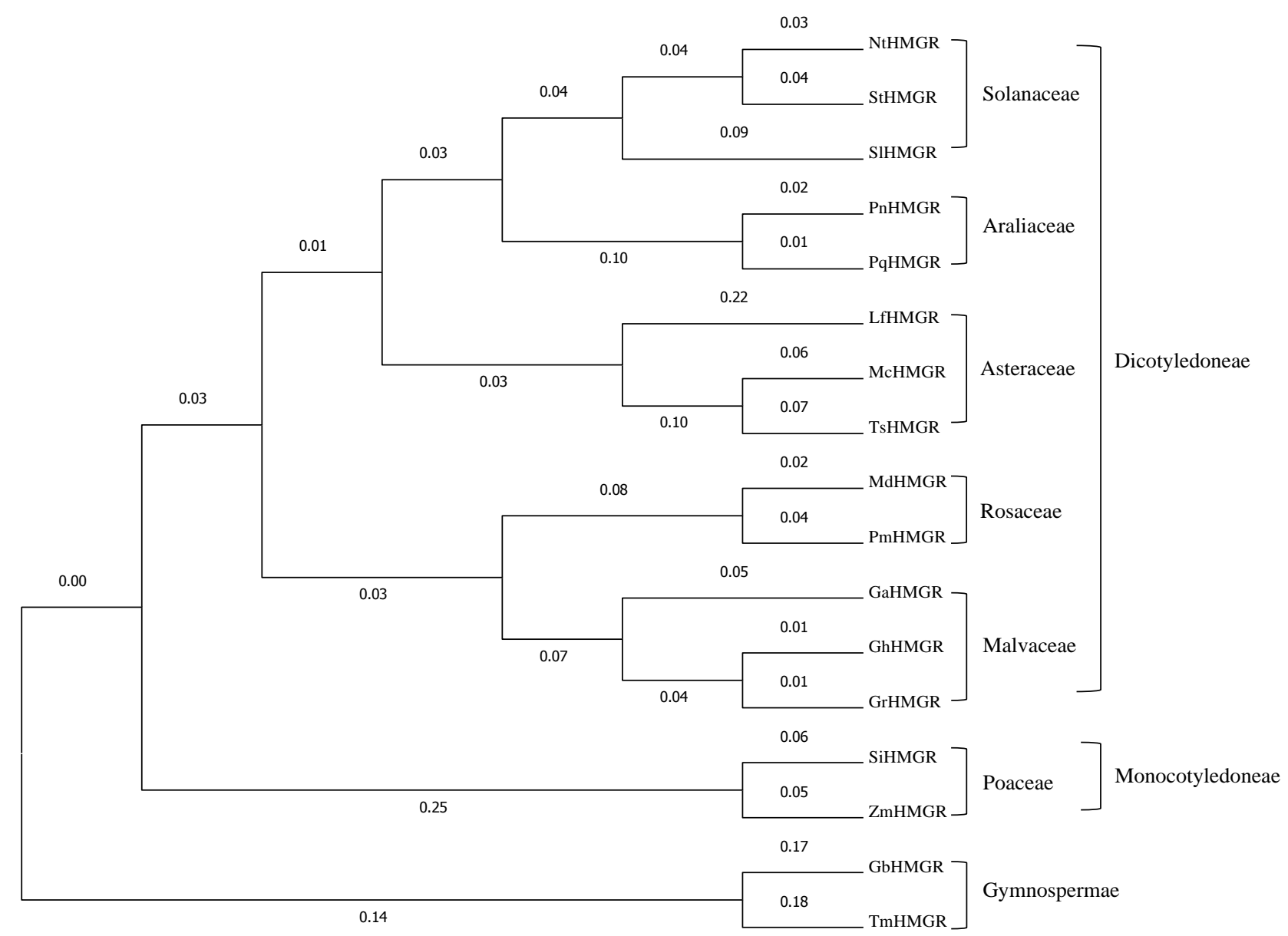

Fig. 3: Phylogenetic tree of HMGR from different species using the Neighbor-joining method. The numbers of nodes represent the percentage of boot strap value obtained from 1000 sampling. Bar 0.01 shows the substitutions per nucleotide position. The species, protein names and GenBank accession number are as following: Solanum lycopersicum: SlHMGR (NP_001234846); Nicotiana tabacum: NtHMGR (XP_016447095.1); Solanum tuberosum: StHMGR (NP_001275084.1); Panax notoginseng: PnHMGR (AKP55621.1); Panax quinquefolius: PqHMGR (NP_001234846); Ligularia fischeri: LfHMGR (ABJ16394.1); Taraxacum kok-saghyz: TsHMGR (AEA92686.1); Malus domestica: MdHMGR (XP_008348952.1); Prunus mume: PmHMGR (XP_008235831.1); Gossypium arboreum: GaHMGR (XP_017636398.1); Gossypium hirsutum: GhHMGR (XP_016728010.1); Gossypium raimondii: GrHMGR (XP_012479904.1); Setaria italica: SiHMGR (XP_004957395.1); Zea mays: ZmHMGR (CAA70440.1); Ginkgo biloba: GbHMGR (AAU89123.1); Taxus x media: TmHMGR (AAQ82685.1).

\section{Expression analysis of $H M G R$ in different tissue of Matricaria chamomilla}

The expression analysis revealed that $M c H M G R$ is ubiquitously expressed in all four different tissues tested but with a strong expression in flowers. Stems recorded the lowest expression level among all the tissue tested (Fig. 4). This result suggested that flowers may a vital tissue for sesquiterpenoid biosynthesis in M. chamomilla. 


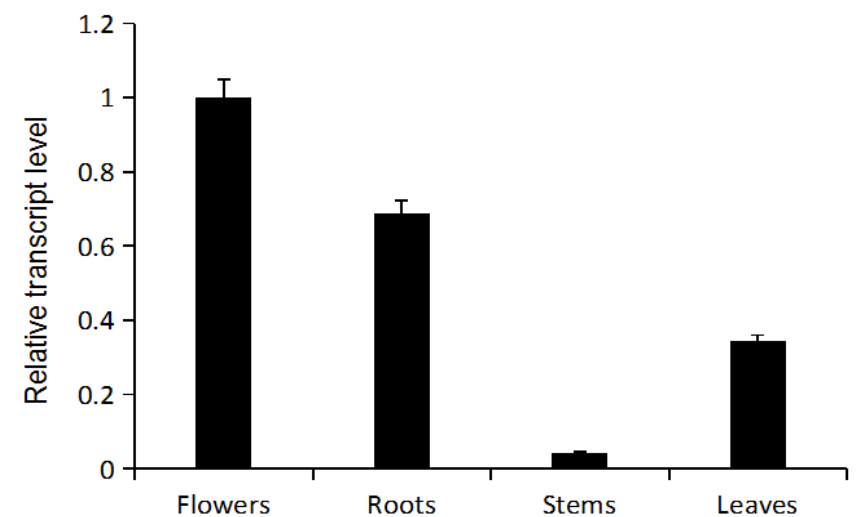

Fig. 4: Real-time PCR for McHMGR gene expression analysis among different tissues of Matricaria chamomilla.

\section{Discussion}

In recent years, there has been a remarkable progress in the understanding of the molecular regulation of isoprenoid biosynthesis in plant (Shen et al., 2006; Wang et al., 2007). 3-Hydroxy-3-methylglutaryl-coenzyme A reductase (HMGR) plays an important role in catalyzing the first committed step of isoprenoids biosynthesis in MVA pathway (Chen et al., 2012). Thus, we attempted to examine the molecular biology of sesquiterpenoid biosynthesis pathways in $M$. chamomilla by cloning and characterization of the full-length cDNA of McHMGR.

In this study, we reported the molecular characterization of $H M G R$ gene from M. chamomilla for the first time. McHMGR is highly similar to HMGR sequences from other species. Multiple sequence alignment analysis of McHMGR also confirmed structural and functional conservation when compared with other plant HMGRs. The phylogenetic tree was constructed considering the HMGRs from plant species other than Asteraceae family to elucidate the evolutionary relationships among the HMGRs which revealed that McHMGR is more distinct and ancient in terms of evolutionary relationship than HMGRs of other plant species.

M. chamomilla is a widely used medicinal plant with great exploitative value for its $\alpha$-bisabolol (Sayadi et al., 2014). M. chamomilla flower is the most commonly used plant part for medicinal purposes (Irmisch et al., 2012). Besides flowers, roots, stems and leaves are also rich in essential oil containing chamazulene, $\alpha$-bisabolol oxide B and $\alpha$-bisabolol oxide A (Hajjaj et al., 2013; Formisano et al., 2015; Roby et al., 2013). Therefore, it is interesting to investigate whether or not McHMGR transcript is possibly correlated with the accumulation of the active compounds in different tissues of $M$. chamomilla. Several studies showed that the expression pattern of $H M G R$ in plant tissues greatly varies across different plants. For example, HMGR is mainly expressed in the node, leaf and stem but is seldom expressed in the root of Centella asiatica (Kalita et al., 2015). However, HMGR exhibited the highest expression level in the leaf and lowest expression level in the stem of Panax quinquefolius (Wu et al., 2012). The expression pattern of McHMGR in M. chamomilla revealed that the gene is expressed in all tissues, but is expressed at higher levels in the flowers. This trend is consistent with the chemical constituent analysis from earlier reports (Farhoudi, 2013), which showed that significant higher contents of the major sesquiterpenoids were observed in the flowers than other parts of M. chamomilla.

\section{Conclusion}

In conclusion, the cloning of $M c H M G R$ provides a foundation for further studies of the biosynthesis of sesquiterpenoids in $M$. chamomilla. In this present investigation, we have successfully cloned and characterized the gene encoding $H M G R$ involved in the biosynthesis of $\alpha$-bisabolol, known as sesquiterpenoids in $M$. chamomilla. Meanwhile, McHMGR is also a committed enzyme in the biosynthetic pathway engineering of active sesquiterpenoids in $M$. chamomilla. Further research will be necessary to clarify the process of catalysis in plants to fully understand the biosynthesis of metabolites. And other relevant genes involved in sesquiterpenoids synthesis in M. chamomilla need to be detected.

\section{Conflict of interest statement}

Authors declare that they have no conflict of interest.

\section{Acknowledgement}

This work was supported by the National Natural Science Foundation of China (31400603), the National Training Programs of Innovation and Entrepreneurship for Undergraduates (104892014043), and the Natural Science Foundation of Hubei Province (2013CFA039).

\section{References}

Akhtar, N., Gupta, P., Sangwan, N. S., Sangwan, R. S., Trivedi, P. K., 2013. Cloning and functional characterization of 3-hydroxy-3-methylglutaryl coenzyme a reductase gene from Withania somnifera: An important medicinal plant. Protoplasma. 250(2), 613-622. 
Attia, M., Kim, S. U., Ro, D. K., 2012. Molecular cloning and characterization of (+)- epi - $\alpha$-bisabolol synthase, catalyzing the first step in the biosynthesis of the natural sweetener, hernandulcin, in Lippia dulcis. Arch. Biochem. Biophys. 527(1), 37-44.

Bonifacio, M., Rigo, A., Guardalben, E., Bergamini, C., Cavalieri, E., Fato, R., 2012. $\alpha$-Bisabolol is an effective proapoptotic agent against bcr-abl+ cells in synergism with imatinib and nilotinib. PLoS One. 7(10), 1172-1178.

Buhaescu, I., Izzedine, H., 2007. Mevalonate pathway: A review of clinical and therapeutical implications. Clin. Biol. 40(9-10), 575-84.

Burnett, R. J., Maldonado-Mendoza, I. E., Mcknight, T. D., Nessler, C. L., 1993. Expression of a 3-hydroxy-3methylglutaryl coenzyme a reductase gene from Camptotheca acuminata is differentially regulated by wounding and methyl jasmonate. Plant. Physiol. 103(1), 41-48.

Chadwick, M., Trewin, H., Gawthrop, F., Wagstaff, C., 2013. Sesquiterpenoids lactones: Benefits to plants and people. Int. J. Mol. Sci. 14(14), 12780-12805.

Chen, X., Wang, X., Li, Z., Kong, L., Liu, G., Fu, J., 2012. Molecular cloning, tissue expression and protein structure prediction of the porcine 3-hydroxy-3-methylglutarylcoenzyme a reductase (HMGR) gene. Gene. 495(2), 170177.

Dai, Z., Cui, G., Zhou, S. F., Zhang, X., Huang, L., 2011. Cloning and characterization of a novel 3-hydroxy-3methylglutaryl coenzyme a reductase gene from Salvia miltiorrhiza, involved in diterpenoid tanshinone accumulation. J. Plant Physiol. 168(2), 148-157.

Farhoudi, R., 2013. Chemical constituents and antioxidant properties of Matricaria recutita and Chamaemelum nobile and essential oil growing wild in the south west of Iran. J. Essen. Oil Bearing Plant. 16(4), 531-537.

Finn, R. D., Clements, J., Eddy, S. R., 2011. Hmmer web server: Interactive sequence similarity searching. Nucl. Acids Res. 39(8), 29-37.

Formisano, C., Delfine, S., Oliviero, F., Tenore, G. C., Rigano, D., Senatore, F., 2015. Correlation among environmental factors, chemical composition and antioxidative properties of essential oil and extracts of chamomile (Matricaria chamomilla L.) collected in Molise (south-central Italy). Ind. Crop. Prod. 63(10), 256-263.

Hajjaj, G., Bounihi, A., Tajani, M., Cherrah, Y., Zellou, A., 2013. Evaluation of CNS activities of Matricaria chamomilla L. essential oil in experimental animals from Morocco. Int. J. Pharm. Pharmaceut. Sci. 5(2), 530-534.

Irmisch, S., Krause, S. T., Kunert, G., Gershenzon, J., Degenhardt, J., Köllner, T. G., 2012. The organ-specific expression of terpene synthase genes contributes to the terpene hydrocarbon composition of chamomile essential oils. BMC Plant. Biol. 12(1), 1-13.

Kalita, R., Patar, L., Shasany, AK., Modi, MK., Sen, P.,2015. Molecular cloning, characterization and expression analysis of 3-hydroxy-3-methylglutaryl coenzyme A reductase gene from Centella asiatica L. Mol. Biol. Rep.
42, 1431-1439.

Kumar, S., Das, M., Singh, A., Ram, G., Mallavarapu, G. R., Ramesh, S., 2001 Composition of the essential oils of the flowers, shoots and roots of two cultivars of Chamomilla recutita. J. Med. Arom. Plant Sci. 23, 617-623.

Liang, Y., Jiang, X., Hu, Q., Li, X., Yin, H., Li, D., 2014. Cloning and characterization of 3-hydroxy-3methylglutaryl-coa reductase (HMGR) gene from Paris fargesii Franch. Ind. J. Biochem. Biophys. 51(3), 201-206.

Liao, Y. L., Feng, X., Huang, X. H., Zhang, W. W., Hua, C., Li, L. L., 2015. Promoter analysis and transcriptional profiling of Ginkgo biloba 3-hydroxy-3-methylglutaryl coenzyme a reductase $(G b H M G R)$ gene in abiotic stress responses. Not. Bot. Horti. Agrobo. 43(1), 25-34.

Liu, Z., Li, X., Simoneau, A. R., Jafari, M., Zi, X., 2006. Isoprenoid biosynthesis in plants: Pathways, genes, regulation and metabolic engineering. J. Biol. Sci. 351(3), 371-374.

Livak, K. J., Schmittgen, T. D., 2001. Analysis of relative gene expression data using real-time quantitative PCR and the 2 $-\Delta \Delta$ CT method. Methods. 25(4), 402-408.

Miziorko, H. M., 2011. Enzymes of the mevalonate pathway of isoprenoid biosynthesis. Arch. Biochem. Biophys. 505(2), 131-143.

Murti, K., Panchal, M. A., Gajera, V., Solanki, J., 2012. Pharmacological properties of Matricaria recutita: A review. Pharmacologia. 3(8), 348-351.

Pirzad, A., Alyari, H., Shakiba, M. R., Zehtabsalmasi, S., 2006. Essential oil content and composition of german chamomile (Matricaria chamomilla L.) at different irrigation regimes. J. Agron. 5(3), 451-455.

Pitera, D. J., Paddon, C. J., Newman, J. D., Keasling, J. D., 2007. Balancing a heterologous mevalonate pathway for improved isoprenoid production in Escherichia coli. Metab. Eng. 9(2), 193-207.

Pleschberger, M., Hildner, F., Rünzler, D., Gelbmann, N., Mayer, H. F., Sleytr, U. B., 2011. Characterization and expression of two cdna encoding 3-hydroxy-3methylglutaryl coenzyme a reductase isoforms in coffee (Coffea arabica L.). Omics J. Integr. Biol. 15(15), 719727.

Raal, A., Orav, A., Püssa, T., Valner, C., Malmiste, B., Arak, E., 2012. Content of essential oil, terpenoids and polyphenols in commercial chamomile (Chamomilla recutita, L. Rauschert) teas from different countries. Food Chem. 131(2), 632-638.

Roby, M. H. H., Sarhan, M. A., Selim, A. H., Khalel, K. I., 2013. Antioxidant and antimicrobial activities of essential oil and extracts of fennel ( foeniculum vulgare, 1.) and chamomile ( Matricaria chamomilla, L.). Ind. Crop. Prod. 44(2), 437-445.

Rui, X., Li, C., Lu, W., Du, J., Wang, Z., Li, J., 2012. 3hydroxy-3-methylglutaryl coenzyme a reductase 1 (hmg1) is highly associated with the cell division during the early stage of fruit development which determines the final fruit size in litchi chinensis. Gene. 498(1), 28-35.

Sayadi, V., Mehrabi, A. A., Saidi, M., Nourollahi, K., 2014. In

T. Tao et al. (2016) / Cloning and Expression Analysis of 3-Hydroxy-3-Methylglutaryl-CoA Reductase Gene from Matricaria chamomilla 
vitro culture and callus induction of chamomile (Matricaria chamomilla L.) explants under different concentrations of plant growth regulators. Int. J. Biosci. 4(10), 206-211.

Shen, G., Pang, Y., Wu, W., Liao, Z., Zhao, L., Sun, X., 2006. Cloning and characterization of a root-specific expressing gene encoding 3-hydroxy-3-methylglutaryl coenzyme a reductase from Ginkgo biloba. Mol. Biol. Rep. 33(2), 117127.

Son, Y. J., Kwon, M., Ro, D. K., Kim, S. U., 2014. Enantioselective microbial synthesis of the indigenous natural product (-)- $\alpha$-bisabolol by a sesquiterpene synthase from chamomile (Matricaria recutita). Biochem. J. 463(2), 239-248.

Su, S. S., Zhang, H. M., Liu, X. Y., Pan, G. F., Ling, S. P., Zhang, X. S., 2015. Cloning and characterization of a farnesyl pyrophosphate synthase from Matricaria recutita L. and its upregulation by methyl jasmonate. Genet. Mol. Res. 14(1), 349-361.
Vranová, E., Coman, D., Gruissem, W., 2013. Network analysis of the MVA and MEP pathways for isoprenoid synthesis. Annu. Rev. Plant Biol. 64(1), 463-476.

Wang, Y., Guo, B., Zhang, F., Yao, H., Miao, Z., Tang, K., 2007. Molecular cloning and functional analysis of the gene encoding 3-hydroxy-3-methylglutaryl coenzyme a reductase from hazel (Corylus avellana L. Gasaway). J. Biochem. Mol. Biol. 40(6), 861-869.

Wu, Q., Chao, S., Chen, S. L., 2012. Identification and expression analysis of a 3-hydroxy-3-methylglutaryl coenzyme A reductase gene from American ginseng. Plant Omics J. 5(4), 414-420.

Xu, F., Ning, Y., Zhang, W., Liao, Y., Li, L., Cheng, H., 2014. An R2R3-MYB transcription factor as a negative regulator of the flavonoid biosynthesis pathway in Ginkgo biloba. Funct. Integr. Genomic. 14(1), 177-189.

Zhao, L., Chang, W., Xiao, Y., Liu, H., Liu, P., 2012. Methylerythritol phosphate pathway of isoprenoid biosynthesis. Annu. Rev. Biochem. 82(1), 497-530.

\section{How to cite this article:}

Tao, T., Liu, X., Chang, J., Xu, F., 2016. Cloning and expression analysis of 3-hydroxy-3-nethylglutaryl-CoA reductase gene from Matricaria chamomilla. Int. J. Curr. Res. Biosci. Plant Biol. 3(11), 9-16.

doi: http://dx.doi.org/10.20546/ijcrbp.2016.311.002 